Eszter Jávorszky*, Vincent Morinière, Andrea Kerti, Eszter Balogh, Henriett Pikó, Sophie Saunier, Veronika Karcagi, Corinne Antignac and Kálmán Tory*

\title{
QMPSF is sensitive and specific in the detection of NPHP1 heterozygous deletions
}

DOI 10.1515/cclm-2016-0819

Received September 12, 2016; accepted November 8, 2016; previously published online December 21, 2016

\begin{abstract}
Background: Nephronophthisis, an autosomal recessive nephropathy, is responsible for $10 \%$ of childhood chronic renal failure. The deletion of its major gene, $N P H P 1$, with a minor allele frequency of $0.24 \%$ in the general population, is the most common mutation leading to a monogenic form of childhood chronic renal failure. It is challenging to detect it in the heterozygous state. We aimed to evaluate the sensitivity and the specificity of the quantitative multiplex PCR of short fluorescent fragments (QMPSF) in its detection.

Methods: After setting up the protocol of QMPSF, we validated it on 39 individuals diagnosed by multiplex ligation-dependent probe amplification (MLPA) with normal NPHP1 copy number $(\mathrm{n}=17)$, with heterozygous deletion ( $n=13$, seven parents and six patients), or with homozygous deletion $(n=9)$. To assess the rate of the deletions that arise from independent events, deleted alleles were haplotyped.
\end{abstract}

*Corresponding authors: Eszter Jávorszky and Kálmán Tory, MTASE Lendulet Nephrogenetic Laboratory, Budapest, Hungary; and 1st Department of Pediatrics, Semmelweis University, Budapest, Hungary, E-mail: javorszky.eszter@med.semmelweis-univ.hu (Eszter Jávorszky); tory.kalman@med.semmelweis-univ.hu (Kálmán Tory)

Vincent Morinière: Assistance Publique-Hôpitaux de Paris, Department of Genetics, Necker Hospital, Paris, France

Andrea Kerti: 1st Department of Pediatrics, Semmelweis University, Budapest, Hungary

Eszter Balogh: MTA-SE Lendulet Nephrogenetic Laboratory, Budapest, Hungary; and 1st Department of Pediatrics, Semmelweis University, Budapest, Hungary

Henriett Pikó and Veronika Karcagi: National Institute of Environmental Health, Department of Molecular Genetics and Diagnostics, Budapest, Hungary

Sophie Saunier: Inserm UMR 1163, Laboratory of Hereditary Kidney Diseases, Paris, France; and Paris Descartes University - Sorbonne Paris Cité, Imagine Institute, Paris, France

Corinne Antignac: Assistance Publique-Hôpitaux de Paris, Department of Genetics, Necker Hospital, Paris, France; Inserm UMR 1163, Laboratory of Hereditary Kidney Diseases, Paris, France; and Paris Descartes University - Sorbonne Paris Cité, Imagine Institute, Paris, France
Results: The results of QMPSF and MLPA correlated perfectly in the identification of 76 heterozygously deleted and 56 homozygously deleted exons. The inter-experimental variability of the dosage quotient obtained by QMPSF was low: control, 1.05 (median; range, 0.86-1.33, $\mathrm{n}=102$ exons); heterozygous deletion, $0.51(0.42-0.67, \mathrm{n}=76$ exons); homozygous deletion, 0 ( $0-0, \mathrm{n}=56$ exons). All patients harboring a heterozygous deletion were found to carry a hemizygous mutation. At least 15 out of 18 deletions appeared on different haplotypes and one deletion appeared de novo.

Conclusions: The cost- and time-effective QMPSF has a $100 \%$ sensitivity and specificity in the detection of $N P H P 1$ deletion. The potential de novo appearance of NPHP1 deletions makes its segregation analysis highly recommended in clinical practice.

Keywords: copy number variation; deletion; nephronophthisis; NPHP1; quantitative multiplex PCR of short fluorescent fragments (QMPSF).

\section{Introduction}

Nephronophthisis is an autosomal recessive kidney disorder leading to interstitial fibrosis and tubular basement membrane alterations. It is a common cause of childhood end-stage renal disease, responsible for $10 \%$ of the cases. Its classical symptoms, polyuria and polydipsia, are typically not severe enough for the parents to seek medical help. Therefore, when no extra-renal involvement is associated, it typically remains unrecognized till the onset of end-stage renal disease [1]. Genetically, it is highly heterogeneous with 20 genes identified thus far [2]. Among them, NPHP1 (OMIM \#256100) is the most frequently mutated due to a recurrent deletion involving the entire gene [3]. This deletion results from an unequal recombination between two directly oriented low-copy repeats, the size of which $(\sim 45 \mathrm{~kb})$ has not yet allowed the identification of the breakpoint [4]. This mechanism makes the NPHP1 deletion the most common mutation in monogenic forms of childhood chronic renal failure with an allele frequency of $0.24 \%$ in the general population [5]. 
In the majority of the patients, the NPHP1 deletion is present in the homozygous state making its detection by PCReasily feasible [6]. Nevertheless, when trans-associated to a point mutation or in carrier parents and siblings, its detection in the heterozygous state may be challenging. As the localization of the breakpoint is unknown, a breakpoint-spanning PCR is not feasible. Therefore, in clinical practice and even in medical reports, the detection of the heterozygous deletion is either neglected or necessitates labor-intensive and expensive methods such as comparative genomic hybridization, fluorescent in situ hybridization or multiplex ligation-dependent probe amplification (MLPA) [7-9].

The time- and cost-effective method of quantitative multiplex PCR of short fluorescent fragments (QMPSF) has been widely used in the identification of copy number variations (CNV) [10-18]. We adapted it for the NPHP1 deletion screening, and here we show its perfect sensitivity and specificity, and the importance of the screening in clinical practice.

\section{Materials and methods}

\section{Patients}

Forty individuals from 28 families (31 patients and 9 parents), diagnosed between 2010 and 2015 at the 1st Department of Pediatrics, Semmelweis University Budapest with potential NPHP1-associated nephronophthisis - i.e. juvenile nephronophthisis, either isolated or with mild Joubert syndrome or late-onset retinopathy [19] - were investigated. Two families were multiplex with two and three affected children. Two families were consanguineous. All families gave informed consent, and the study was approved by the Local Research Ethic Committee (TUKEB 6569-0/2010-1018EKU).

\section{DNA extraction}

DNA was extracted based on proteinase $\mathrm{K}$ digestion followed by high-sodium chloride treatment to precipitate proteins. DNA concentration was measured by NanoDrop ${ }^{\mathrm{TM}} 1000$ Spectrophotometer (Thermo Fisher Scientific, Waltham, MA, USA) and was diluted with TE buffer (10 mM Tris-HCl pH 8.2, 1 mM EDTA) to achieve a final concentration of $10 \mathrm{ng} / \mu \mathrm{L}$ for QMPSF and $20 \mathrm{ng} / \mu \mathrm{L}$ for MLPA.

\section{Multiplex ligation-dependent probe amplification assay}

All samples were analyzed by the NPHP1-specific reagent kit, covering all 20 exons (SALSA MLPA P387 NPHP1 probemix; MRC-Holland, Amsterdam, The Netherlands), according to the manufacturer's instructions, with 100 ng genomic DNA. Fragment analysis was performed on ABI 3500 Genetic Analyzer (Applied Biosystems, Foster City, CA, USA), and the results were evaluated using Peak Scanner Software (Thermo Fischer Scientific) and Coffalyser (MRC-Holland).

\section{Validation of the identified deletions}

All NPHP1 deletions - identified by MLPA - were confirmed by a second method in the affected individuals. Homozygous deletions were validated by PCR as previously described [6]. Patients with heterozygous deletion were screened for point mutations in the coding regions and the splicing junctions of NPHP1 (NM_000272) by Sanger sequencing [4]. The hemizygous state of the point mutations was confirmed by haplotype and segregation analysis. A partial 3' deletion was confirmed by array CGH.

\section{Quantitative multiplex PCR of short fluorescent fragments (QMPSF)}

All samples were investigated by QMPSF. Short, 120- to 321-bp-long fragments of six NPHP1 (2q13) exons [3, 6, 9, 12, 15, 19] together with two control regions of HSD17B3 (9q22.32) and USH2A (1q41) were amplified by multiplex PCR (Table 1, Figure 1).

The conditions were set up in a patient with a heterozygous deletion and in three control subjects, two of whom was diagnosed with an HNF1B deletion and one with amyloidosis, who was also confirmed by MLPA to have a normal NPHP1 copy number. The multiplex PCR was performed in $30 \mu \mathrm{L}$ of reaction master mixture containing $0.2 \mathrm{mmol} / \mathrm{L}$ of each dNTP, $1.5 \mathrm{mmol} / \mathrm{L} \mathrm{MgCl}_{2}, 0.6 \mathrm{U}$ Immolase DNA polymerase (ImmoMix ${ }^{\mathrm{TM}}$, Bioline Inc.), and 0.1-0.4 $\mu \mathrm{mol} / \mathrm{L}$ of each primer as specified in Table 1. Twenty-nanogram genomic DNA $(2 \mu \mathrm{L})$ was added to yield a final volume of $32 \mu \mathrm{L}$. The initial denaturation $\left(95^{\circ} \mathrm{C}\right.$ for $12 \mathrm{~min}$ ) was followed by 26 cycles of amplification $\left(95^{\circ} \mathrm{C}\right.$ for $45 \mathrm{~s}, 60^{\circ} \mathrm{C}$ for $45 \mathrm{~s}, 72^{\circ} \mathrm{C}$ for $\left.45 \mathrm{~s}\right)$ and a final extension $\left(72^{\circ} \mathrm{C}\right.$ for $10 \mathrm{~min}$ ). This limited number of cycles stops the amplification in the exponential phase keeping the amount of the PCR product proportional to the original amount of the targeted sequence, thus allowing the quantification of the target sequence relative to the control regions. All forward primers were labeled with 6-FAM fluorophore and universal extensions were added to the $5^{\prime}$ end of both forward and reverse primers $[13,16,20]$. Fragment analysis was carried out in the same way as for MLPA.

Four controls (three negative and one with a known heterozygous deletion identified by MLPA) were run in each assay. Results of QMPSF were analyzed only if the peak height of both HSD17B3 and USH2A control regions were above 2000 relative fluorescence units. To determine the gene dosage for each amplified region, dosage quotient (DQ) was calculated: the ratio of the target peak area and the average of HSD17B3 and USH2A peak areas was determined for each patient and was subsequently divided by the controls' average ratio. This gives a theoretical DQ of 1.0 for two NPHP1 copies and 0.5 for a heterozygous deletion [10,12, 21, 22]. Following the criteria of the MLPA protocol, we considered a DQ value between 0.3 and 0.7 to reflect a heterozygous deletion and a DQ value between 0.7 and 1.3 to reflect a normal copy number [23, 24]. 
Table 1: NPHP1-QMPSF primer sequences.

\begin{tabular}{|c|c|c|c|}
\hline Primer & Primer sequence & Product length, bp & Concentration, $\mu \mathrm{mol} / \mathrm{L}$ \\
\hline NPHP1 3F & СCTGCTCAACGACTGAATGA & 136 & 0.1 \\
\hline NPHP1 3R & TCTATTGCCTGCTTTAACTGGA & & \\
\hline NPHP1 6F & CGGGTGATAGGGAAGCTTTTT & 225 & 0.2 \\
\hline NPHP1 6R & TCCACCAACCATCAGGTTTT & & \\
\hline NPHP19F & TGCAGAAACATGACTGAAAACA & 241 & 0.4 \\
\hline NPHP1 9R & TGTGTTTTGCCTGTGACAGAA & & \\
\hline NPHP1 12F & AGCCACATGGCAACCTAAAA & 200 & 0.4 \\
\hline NPHP1 12R & CAATGTCCTCAAAGAACACCAA & & \\
\hline NPHP1 15F & ACCTCATGGAGGGATCAACA & 321 & 0.4 \\
\hline NPHP1 15R & GCTACCTCTCAGATGCTTCTATTTG & & \\
\hline NPHP1 19F & TTTCTGTTTGCTCTTTAGAGTTCG & 120 & 0.2 \\
\hline NPHP1 19R & GCAAATATGGAGTTCAGTGTGG & & \\
\hline$H S D 17 B 311 \mathrm{~F}$ & AAGGCTGCTCCTGACACACT & 186 & 0.2 \\
\hline HSD17B3 11R & ССTCCATCTTCAGCGGACTA & & \\
\hline USH2A 41F & CCTTTCACCAGAGTCCCAGA & 273 & 0.2 \\
\hline USH2A 41R & CCATGGGCTAAGAGCAGAAG & & \\
\hline
\end{tabular}

Primers of control regions are in bold.

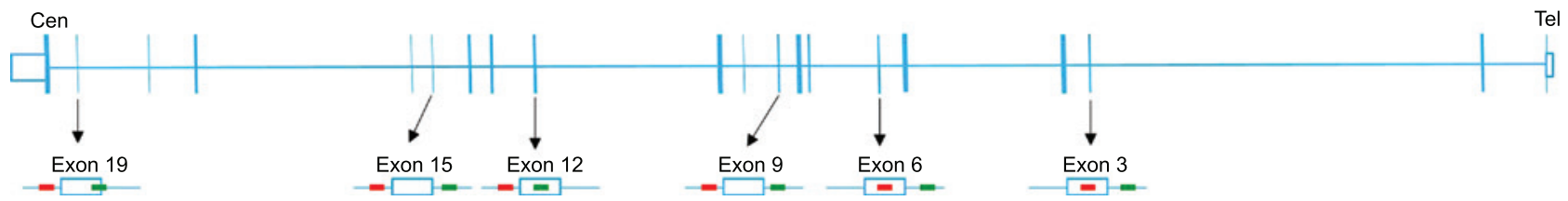

Figure 1: The position of the NPHP1-QMPSF primers (2q13, ENST00000393272.3) as shown by green (forward) and red lines (reverse primers). Cen, centrome; Tel, telomere.

\section{Haplotype analysis}

The D2S293, D2S340, D2S1893, D2S160, and D2S363 microsatellite markers - located outside the NPHP1 deletion - were used for haplotype analysis as described previously [25-27].

\section{CGH array}

CGH array analysis was performed in a patient who was compound heterozygous for two different deletions. A high-resolution genomic scan using ISCA plus design array of Nimblegen-Roche containing 1.4 M probes per subarray (assembly GRCh37/hg19) was performed in the DNA sample of the patient. This CGX microarray provides a mean average resolution of approximately $15-20 \mathrm{~kb}$. Array CGH analysis was performed according to the manufacturer's protocol. The CGH protocol involves independent labeling of the patient (test DNA) and the reference genomic DNA (Human Genomic DNA, Promega, Madison, WI, USA) with Cy3 and Cy5 dyes using a NimbleGen Dual-Color DNA Labeling Kit (Roche-NimbleGen Inc.). Co-hybridization of these DNAs to a NimbleGen CGH array were performed for $72 \mathrm{~h}$ at $42^{\circ} \mathrm{C}$. The subarrays were scanned on NimbleGen MS 200 microarray scanner and data were extracted and analysed using NimbleScan, SignalMap, and Deva 1.1 softwares (Roche NimbleGen Inc.).

\section{Results}

\section{High rate of NPHP1 deletion in juvenile nephronophthisis}

Out of the 31 patients with juvenile nephronophthisis, we found $16(52 \%)$ patients (14/28 families) to carry NPHP1 deletion (c.(?_-1)_( ${ }^{\star} 1 \_$? )del) on at least one allele by MLPA (Table 2). Nine unrelated patients were homozygous and five patients from three families were heterozygous for the deletion. The homozygous deletion was confirmed in all cases by PCR and all five patients with a heterozygous deletion were found to carry a hemizygous point mutation, two of which (c.489delT, p.Phe163Leufs ${ }^{\star} 19$, and c.656C > A, p.Ser219^) are novel (Figure 2).

Two more unrelated patients were compound heterozygous for the classical whole-gene deletion and a novel 3' partial deletion (c.(1810+1_1811-1)_( $\left.{ }^{\star} 1 \_?\right)$ del) involving the last three [18-20] exons. The latter was confirmed by array CGH in one of the two patients (hg19 
Table 2: NPHP1 mutations of the patients.

\begin{tabular}{|c|c|c|}
\hline Families & Paternal allele & Maternal allele \\
\hline F10, F12, F15, F63, F67, F70, F84, F97, F158 & del $^{\mathrm{a}}$ & del $^{a}$ \\
\hline F36 & c.84_87delTTCT, p.Ser29Argfs* 4 & del $^{a}$ \\
\hline F38 & c.489delT, p.Phe163Leufs*19 & del $^{\mathrm{a}}$ \\
\hline F249 & c.656C $>A$, p.Ser219*; del ${ }^{b}$ & \\
\hline F106 & Exon $18-20$ deletion ${ }^{c}$ & del $^{\mathrm{a}}$ \\
\hline F203 & del $^{\mathrm{a}}$ & Exon $18-20$ deletion $^{c}$ \\
\hline
\end{tabular}

adel.: c.(?_-1)_(`1_?)del, bunknown segregation, 'c.(1810+1_1811-1)_(*1_?)del or g.(110850451_110862515)_(110888028_110889217)del.

c.84 87delTTCT

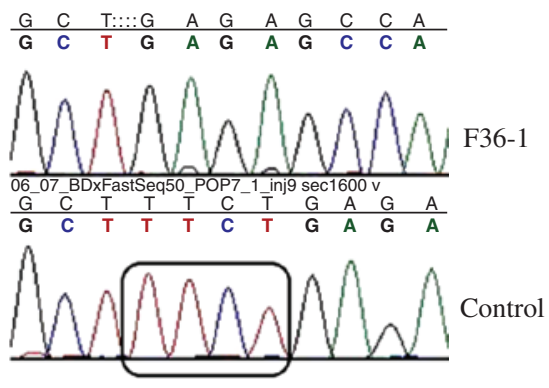

c.489delT
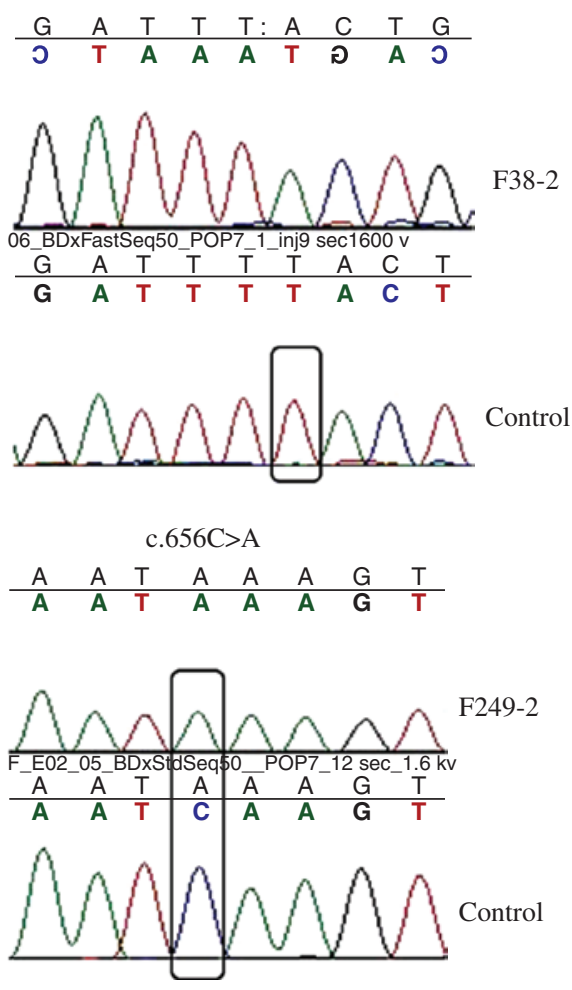

Figure 2: Chromatograms of the hemizygous mutations.

chr2: g.(110850451_110862515)_(110888028_110889217) del, F106-1, Figure 3) and the second patient (F203-2) was shown to carry the same haplotype on the $3^{\prime}$ partially deleted NPHP1 allele, reflecting a founder effect (Figure 4).

\section{NPHP1 deletions typically result from independent events}

Out of the 16 deletions of non-consanguineous families, we found one deletion to appear de novo, on the paternal allele (F84). The biological paternity was confirmed by haplotype analysis (Figure 5).

Furthermore, among the 18 haplotypes with whole gene deletion (taking into account the alleles of the two consanguineous families as a single allele), we found at least 15 different alleles indicating a high rate of independent deletions, which is in accordance with previous results and contrasts the founder $3^{\prime}$ partial deletion (Figure 4) [4, 28].

\section{Intra-experimental variability of NPHP1-QMPSF is low}

We found the intra-experimental variability of NPHP1QMPSF - through investigating a control subject and a patient with a heterozygous deletion in ten parallel measurements - to be low: $\mathrm{DQ}_{\text {control }}=0.98$ (median; quartiles, 0.96-1.01; range, 0.86-1.09); $\mathrm{DQ}_{\text {het.del }}=0.51$ (median; quartiles, 0.50-0.53; range, 0.45-0.57).

\section{The specificity and sensitivity of QMPSF are perfect in the detection of an NPHP1 deletion}

There was a clear-cut difference in the DQ values of 17 control subjects, 13 parents and patients with heterozygous deletion, and 9 patients with homozygous deletion in all six exons tested (Figures 6 and 7), as also indicated by the overall values: $\mathrm{DQ}_{\text {control }}=1.05$ (median; quartiles, 0.99-1.08; range, 0.86-1.33); $\mathrm{DQ}_{\text {het.del }}=0.51$ (median; quartiles, 0.500.54; range: 0.42-0.67); $\mathrm{DQ}_{\text {hom.del }}=0$ (median; range, $0-0$ ).

The two patients with compound heterozygous deletions were properly found to be heterozygous for exons 


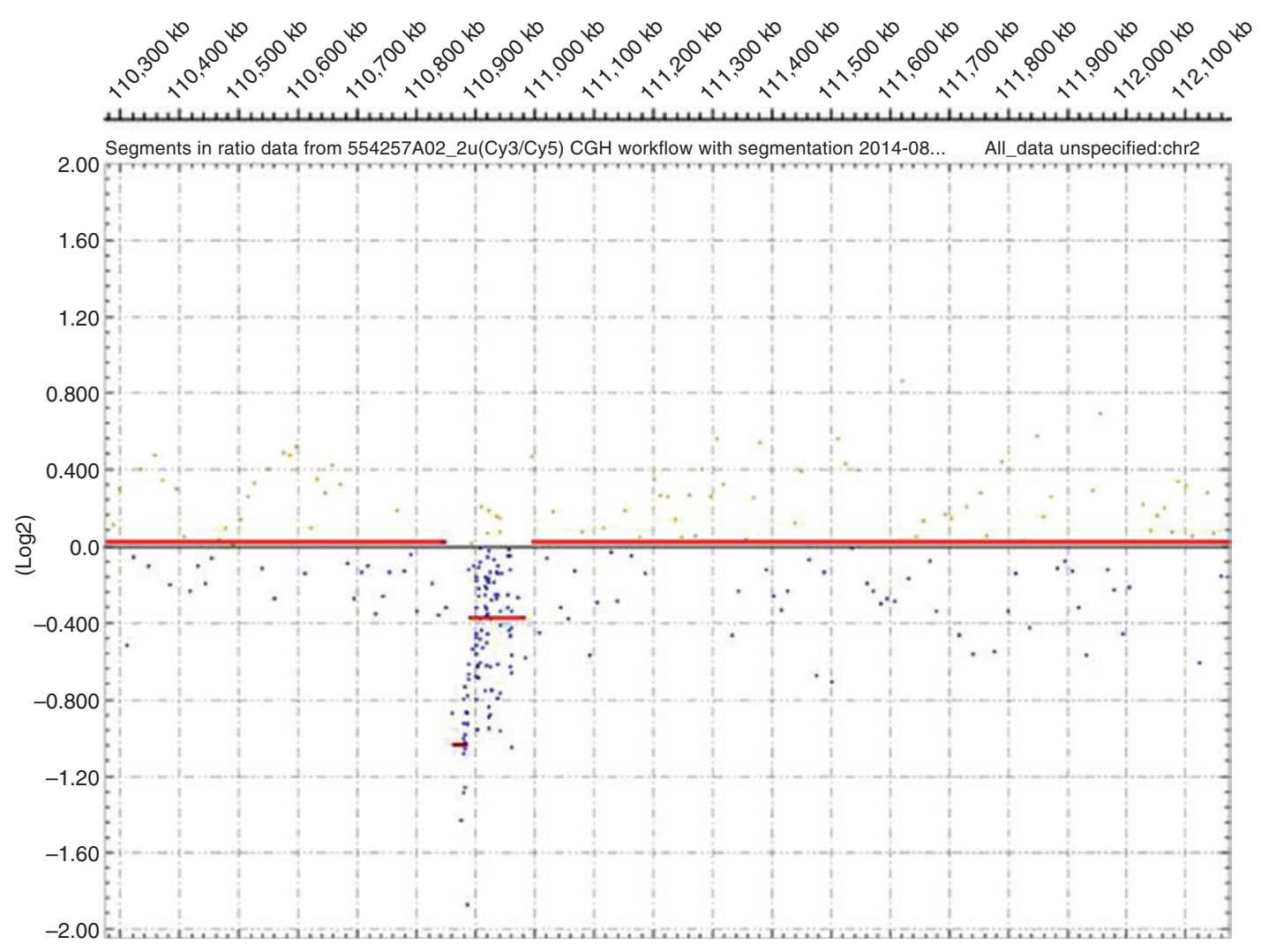

Figure 3: Array CGH of patient F106-1.

Compound heterozygous deletion in the NPHP1 gene: while both alleles are deleted (score -1.05) in the region chr2:g.110862515_110888028 (on Assembly GRCh37), a heterozygous deletion (score - 0.38) is found in the region g.110888028_110985511. This reflects the transassociation of the classical whole gene deletion (g.(110850451_110862515)_(110985511_110996482)del) and a partial 3' deletion (g.(110850451_110862515)_(110888028_110889217)del), and defines the 5' breakpoint of the partial deletion in intron 17.

\begin{tabular}{|c|c|c|c|c|c|c|c|c|c|c|c|c|c|c|c|c|c|c|c|c|c|c|c|c|c|}
\hline \multirow{3}{*}{$\begin{array}{l}\text { Marker } \\
\text { D2S293 }\end{array}$} & \multirow{3}{*}{$\begin{array}{c}\text { gen. pos. } \\
\text { (GRCh37) } \\
107.3\end{array}$} & \multicolumn{24}{|c|}{ Patients } \\
\hline & & \multicolumn{2}{|c|}{ F10-2 } & \multicolumn{2}{|c|}{ F12-2 } & \multicolumn{2}{|c|}{ F15-1 } & \multicolumn{2}{|c|}{ F36-1 } & \multicolumn{2}{|c|}{ F36-2 } & \multicolumn{2}{|c|}{ F67-2' } & \multicolumn{2}{|c|}{ F70-2 } & \multicolumn{2}{|c|}{ F84-2 } & \multicolumn{2}{|c|}{ F97-2 } & \multicolumn{2}{|c|}{ F106-1 } & \multicolumn{2}{|c|}{ F158-6' } & \multicolumn{2}{|c|}{ F203-2 } \\
\hline & & 3 & 6 & 3 & 10 & 2 & 11 & 1 & & 5 & 2 & 4 & 4 & 2 & 9 & 2 & 7 & $3 / 7$ & $3 / 7$ & 7 & 4 & 6 & 6 & 3 & 4 \\
\hline D2S340 & 108.9 & 4 & 3 & 3 & 5 & 3 & 6 & 5 & 3 & 4 & 5 & 3 & 3 & 2 & 3 & 5 & 5 & 1 & 5 & 3 & 4 & 3 & 3 & 4 & 4 \\
\hline D2S1893 & 109.8 & 1 & 9 & 11 & 6 & 9 & 6 & 9 & 9 & 6 & 10 & 9 & 9 & 9 & 9 & 10 & 10 & 6 & 9 & 10 & 6 & 10 & 10 & 11 & 6 \\
\hline NPHP1 & 110.9 & Del & Del & Del & Del & Del & Del & Del & pm & Del & pm & Del & Del & Del & Del & Del & Del & Del & Del & Del & 'de & Del & Del & Del 3 & 3'del \\
\hline D2S160 & 113.0 & 5 & 6 & 9 & 6 & 6 & 7 & 4 & 2 & 5 & 5 & 6 & 6 & 2 & 5 & 6 & 5 & 5 & 7 & 1 & 3 & 6 & 6 & 5 & 6 \\
\hline D2S363 & 117.4 & 4 & 1 & 2 & 2 & $1 / 2$ & $1 / 2$ & 2 & 5 & $1 / 2$ & $1 / 2$ & 2 & 2 & 1 & 2 & 2 & 2 & 1 & 1 & 2 & 1 & 4 & 4 & $2 / 3$ & $2 / 3$ \\
\hline
\end{tabular}

Figure 4: Haplotype of patients with NPHP1 deletion.

Del: whole gene deletion (c.(?_-1)_(*1_?)del), 3’del: exon 18-20 deletion (c.(1810+1_1811-1)_( $\left.{ }^{\star} 1 \_?\right)$ del), pm, point mutation; a, products of consanguineous marriages. Similar haplotypes are highlighted by the same gray shade.

$3,6,9,12$, and 15 deletions and to be deleted on both alleles in exon 19. Thus, with the exception of a DQ of 1.33 erroneously suggesting a duplication for exon 3 in a patient with a normal copy number (F2-1), results for all exons of all patients correlated perfectly with the MLPA results. Thus, all and only the 76 heterozygously deleted exons were identified by QMPSF to be deleted in the heterozygous state, giving $100 \%$ sensitivity and
$100 \%$ specificity for the identification of a heterozygous deletion.

\section{Discussion}

The NPHP1 gene deletion is one of the most common mutations leading to a severe monogenic disorder: it is only 


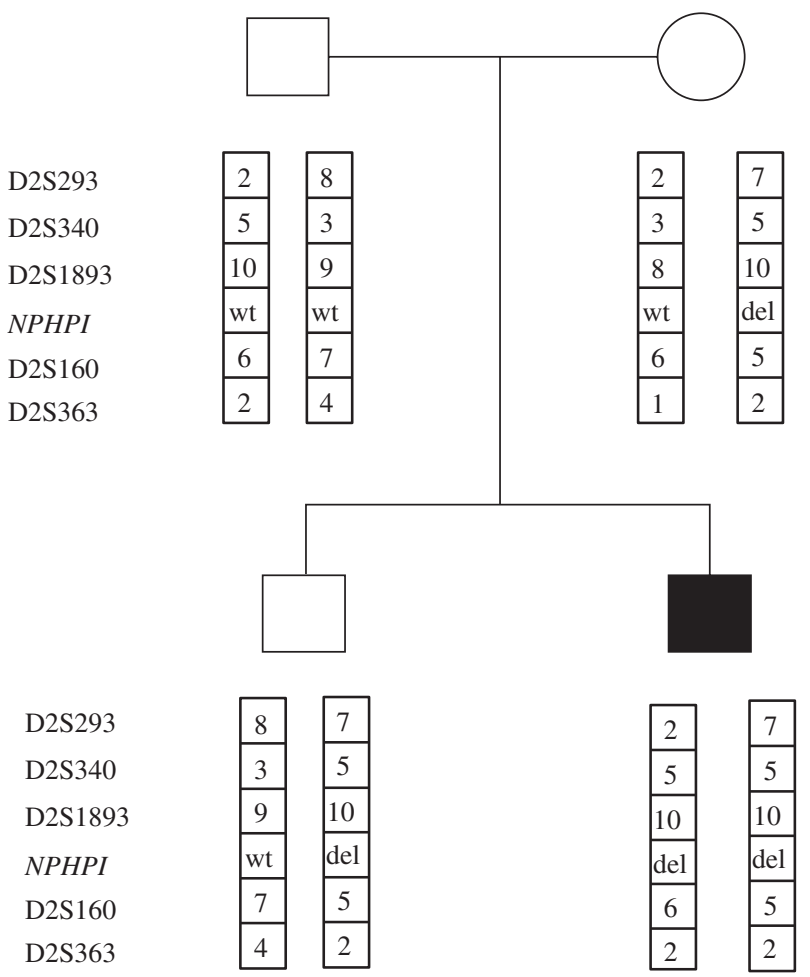

Figure 5: Haplotype analysis of the family F84 with a de novo deletion.

We found no recombination in the NPHP1 region between the two paternal chromosomes suggesting that the deletion resulted from an intrachromosomal loop formation.

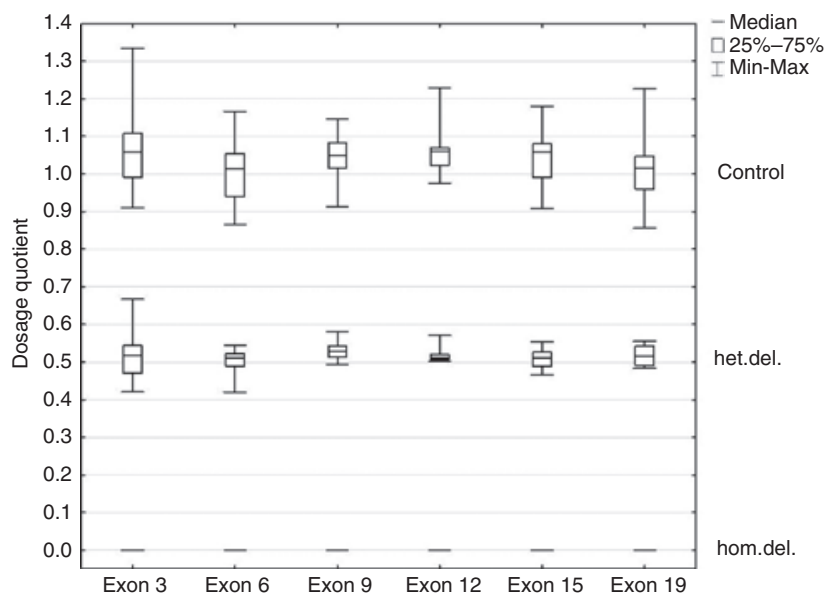

Figure 6: Dosage quotient of NPHP1 exons determined by QMPSF in patients and control subjects.

four times less frequent than the CFTR p.Phe508del mutation, and twice as frequent as the NPHS2 p.R138Q mutation in the European population [5, 9, 29]. As such, to the best of our knowledge, it is the most common mutation leading to chronic renal failure in childhood. In accordance with its frequent causal role in nephronophthisis, we

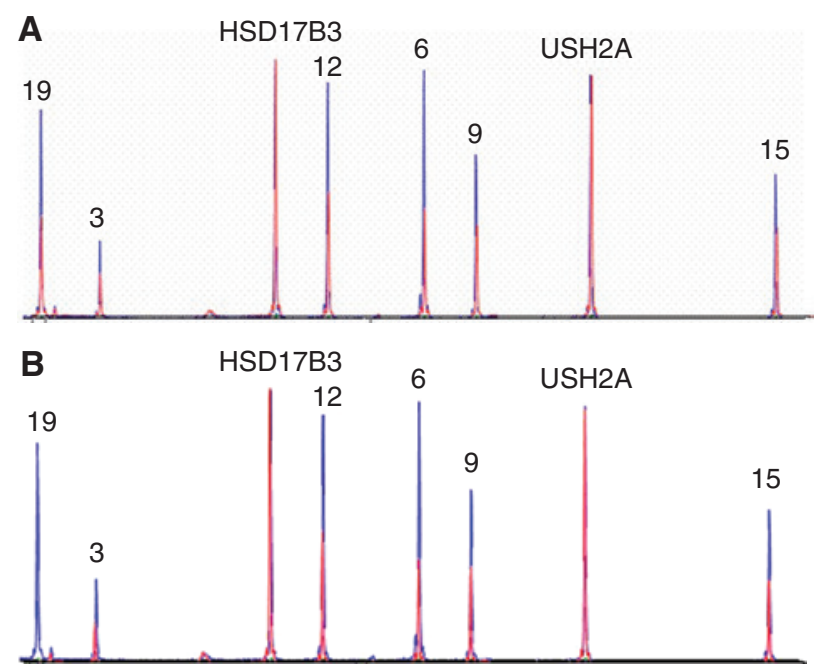

Figure 7: Electropherograms of either a patient with heterozygous deletion (A) or a patient (F106-1) with compound heterozygous deletion (B) (in red), superimposed on the electropherograms of a control subject (in blue).

While control regions (HSD17B3 and USH2A) show a similar peak height in patients and controls, heterozygous deletion reduces the peak height by half and a deletion on both alleles (B, patient F106-1, exon 19) abolishes it.

found it in the families with juvenile nephronophthisis in the homozygous and heterozygous state in $32 \%$ and $18 \%$, respectively. Such a high NPHP1 mutation rate was found in other single center cohorts $[9,30,31$, while a lower rate was reported in worldwide cohorts [2], probably due to the less stringent clinical inclusion criteria. Although the majority of the patients carry the NPHP1 deletion in the homozygous state, we found one third of the patients with NPHP1 deletions to be compound heterozygous for the whole gene deletion and either a point mutation or a partial deletion. A similar ratio was reported in other cohorts [9, 32], making the identification of NPHP1 deletion in the heterozygous state important. One could argue that sequencing of NPHP1 would allow the identification of the trans-associated hemizygous mutations and thus it is useless to identify the NPHP1 deletion in the heterozygous state. Nevertheless, differentiation of the homozygous and hemizygous state of the trans-associated mutations is possible only if parental DNA samples are available, and even if they are, it is difficult or even impossible to conclude whether the deletion is inherited or appeared de novo. This latter seems to be crucial in case of the NPHP1 gene deletion, which - in contrast to the vast majority of the recessive mutations or the novel 3 ' partial deletion - is not a founder mutation, but arises from independent events [4, 28]. This is very well reflected in our study by the detection of a de novo deletion and by the very different haplotypes of the deleted alleles. 
Even among families with homozygous deletion, only the consanguineous families carried the same haplotype on the two alleles. The independent origin of the deletions also explains its similarly high frequency in different geographic area [9, 30, 31]. Two mechanisms have been proposed to explain the appearance of an NPHP1 deletion: an unequal recombination secondary to (1) chromosome misalignment following an intrachromosomal loop formation or secondary to (2) unequal crossing over between the two homolog chromosomes leading to a deletion on one and a duplication on the other homologue [4]. We found no crossing over and thus suggest the first mechanism to be causal in the family with the de novo deletion (Figure 5). The de novo appearance dramatically reduces the risk of recurrence among the siblings, but does not abolish it as parental mosaicism was found in $4 \%$ of families with de novo CNV [33]. As the de novo rate of the NPHP1 deletion $-1 / 16$ in the present and $1 / 14$ in a previous study [34] - is not negligible, it is crucial to verify the carrier status of the parents. Furthermore, given the carrier frequency of $\sim 1: 200$ in the general population, one could even consider screening the partners of mutation carriers. All these aspects make the detection of the heterozygous NPHP1 deletion important both for the genetic diagnosis and for genetic counseling. However, the carrier status of the parents has rarely been investigated [35].

Given the frequency of the NPHP1 deletion and the difficulty of its detection by multiple parallel sequencing, its targeted genetic screening should precede any other genetic test once the clinical diagnosis of juvenile nephronophthisis is raised [1]. As QMPSF is $3.5 \times$ less expensive in our setting than MLPA, and replaces the four-step procedure of MLPA by a single one, we tested it for the detection of NPHP1 deletion. The diagnosis of NPHP1 deletion was primarily established by MLPA and confirmed by a second test in all patients. Within this cohort, we found both the specificity and the sensitivity of QMPSF to be $100 \%$ for the detection of both the heterozygous and the homozygous NPHP1 deletion. We also properly identified a compound heterozygous deletion by QMPSF, though our method covers only six out of 20 exons. To achieve such a high sensitivity and specificity, it is essential to properly stop the amplification before the saturation and thus to keep the amount of the PCR product proportional to the original amount of the targeted sequence. A potential disadvantage of our QMPSF method is that it misses those CNVs that are located in-between the targeted six exons. Though no such a small CNV has been reported thus far in NPHP1, they would go undetected by this method. On the other hand, though duplications of $N P H P 1$ are not implicated in the pathogenesis of nephronophthisis and thus could not be investigated, they have been reported in patients with autism [36] and are expected to be detectable by QMPSF.

We thus conclude that NPHP1-QMPSF is perfectly suitable for the detection of the NPHP1 deletion even in the heterozygous state and thus suitable for the clinical diagnosis. As the NPHP1 deletion may occur de novo, the carrier status of the parents is highly recommended to be verified in clinical practice.

Author contributions: All the authors have accepted responsibility for the entire content of this submitted manuscript and approved submission.

Research funding: Financial support for this work was provided by MTA-SE Lendulet Research Grant (LP2015-11/2015) of the Hungarian Academy of Sciences, OTKA K109718 and by the French-Hungarian bilateral project (PHC 34501SM Balaton, Hungarian Grant No. TeT_14_1-2015-0020).

Employment or leadership: None declared.

Honorarium: None declared.

Competing interests: The funding organization(s) played no role in the study design; in the collection, analysis, and interpretation of data; in the writing of the report; or in the decision to submit the report for publication.

\section{References}

1. Salomon R, Saunier S, Niaudet P. Nephronophthisis. Pediatr Nephrol 2009;24:2333-44.

2. Halbritter J, Porath JD, Diaz KA, Braun DA, Kohl S, Chaki M, et al. Identification of 99 novel mutations in a worldwide cohort of 1,056 patients with a nephronophthisis-related ciliopathy. Hum Genet 2013;132:865-84.

3. Saunier S, Calado J, Heilig R, Silbermann F, Benessy F, Morin $\mathrm{G}$, et al. A novel gene that encodes a protein with a putative src homology 3 domain is a candidate gene for familial juvenile nephronophthisis. Hum Mol Genet 1997;6:2317-23.

4. Saunier S, Calado J, Benessy F, Silbermann F, Heilig R, Weissenbach J, et al. Characterization of the NPHP1 locus: mutational mechanism involved in deletions in familial juvenile nephronophthisis. Am J Hum Genet 2000;66:778-89.

5. Coe BP, Witherspoon K, Rosenfeld JA, van Bon BW, Vulto-van Silfhout AT, Bosco P, et al. Refining analyses of copy number variation identifies specific genes associated with developmental delay. Nat Genet 2014;46:1063-71.

6. Konrad M, Saunier S, Heidet L, Silbermann F, Benessy F, Calado J, et al. Large homozygous deletions of the 2 q13 region are a major cause of juvenile nephronophthisis. Hum Mol Genet 1996;5:367-71.

7. Haghighi A, Savaj S, Haghighi-Kakhki H, Benoit V, Grisart B, Dahan K. Identification of an NPHP1 deletion causing adult form of nephronophthisis. Ir J Med Sci 2016;185:589-95.

8. Lindstrand A, Davis EE, Carvalho CM, Pehlivan D, Willer JR, Tsai IC, et al. Recurrent CNVs and SNVs at the NPHP1 locus contribute 
pathogenic alleles to Bardet-Biedl syndrome. Am J Hum Genet 2014;94:745-54.

9. Hildebrandt F, Rensing C, Betz R, Sommer U, Birnbaum S, Imm $A$, et al. Establishing an algorithm for molecular genetic diagnostics in 127 families with juvenile nephronophthisis. Kidney Int 2001;59:434-45.

10. Castellsague E, Gonzalez S, Nadal M, Campos O, Guino E, Urioste $M$, et al. Detection of APC gene deletions using quantitative multiplex PCR of short fluorescent fragments. Clin Chem 2008;54:1132-40.

11. Houdayer C, Gauthier-Villars M, Lauge A, Pages-Berhouet S, Dehainault C, Caux-Moncoutier V, et al. Comprehensive screening for constitutional RB1 mutations by DHPLC and QMPSF. Hum Mutat 2004;23:193-202.

12. Mo ZP, Yu CS, Li YJ, Cao WX, Zeng ZY, Zhan YX, et al. Detection of alpha-globin gene deletion and duplication using quantitative multiplex PCR of short fluorescent fragments. Clin Chem Lab Med 2012;50:649-54.

13. Rouzier C, Chaussenot A, Serre V, Fragaki K, Bannwarth S, Ait-ElMkadem S, et al. Quantitative multiplex PCR of short fluorescent fragments for the detection of large intragenic POLG rearrangements in a large French cohort. Eur J Hum Genet 2014;22:542-50.

14. Saugier-Veber P, Goldenberg A, Drouin-Garraud V, de La Rochebrochard C, Layet V, Drouot N, et al. Simple detection of genomic microdeletions and microduplications using QMPSF in patients with idiopathic mental retardation. Eur J Hum Genet 2006;14:1009-17.

15. Vaurs-Barriere C, Bonnet-Dupeyron MN, Combes P, GauthierBarichard F, Reveles XT, Schiffmann R, et al. Golli-MBP copy number analysis by FISH, QMPSF and MAPH in 195 patients with hypomyelinating leukodystrophies. Ann Hum Genet 2006;70(Pt 1):66-77.

16. Bellanne-Chantelot C, Clauin S, Chauveau D, Collin P, Daumont M, Douillard C, et al. Large genomic rearrangements in the hepatocyte nuclear factor-1beta (TCF2) gene are the most frequent cause of maturity-onset diabetes of the young type 5 . Diabetes 2005;54:3126-32.

17. Jardin F, Ruminy P, Kerckaert JP, Parmentier F, Picquenot JM, Quief S, et al. Detection of somatic quantitative genetic alterations by multiplex polymerase chain reaction for the prediction of outcome in diffuse large B-cell lymphomas. Haematologica 2008;93:543-50.

18. Ferreira MC, Dorboz I, Rodriguez D, Boespflug TO. Screening for GFAP rearrangements in a cohort of Alexander disease and undetermined leukoencephalopathy patients. Eur J Med Genet 2015;58:466-70.

19. Tory K, Lacoste T, Burglen L, Moriniere V, Boddaert N, Macher MA, et al. High NPHP1 and NPHP6 mutation rate in patients with Joubert syndrome and nephronophthisis: potential epistatic effect of NPHP6 and AHI1 mutations in patients with NPHP1 mutations. J Am Soc Nephrol 2007;18:1566-75.

20. Nicolas G, Rovelet-Lecrux A, Pottier C, Martinaud O, Wallon D, Vernier L, et al. PDGFB partial deletion: a new, rare mechanism causing brain calcification with leukoencephalopathy. J Mol Neurosci 2014;53:171-5.

21. Gouas L, Goumy C, Veronese L, Tchirkov A, Vago P. Gene dosage methods as diagnostic tools for the identification of chromosome abnormalities. Pathol Biol (Paris) 2008;56:345-53.
22. Hyon C, Mansour-Hendili L, Chantot-Bastaraud S, Donadille $B$, Kerlan V, Dode C, et al. Deletion of CPEB1 gene: a rare but recurrent cause of premature ovarian insufficiency (POI). J Clin Endocrinol Metab 2016;101:2099-104.

23. Kiehntopf M, Varga RE, Koch HG, Beetz C. A homemade MLPA assay detects known CTNS mutations and identifies a novel deletion in a previously unresolved cystinosis family. Gene 2012;495:89-92.

24. Zhang X, Xu Y, Liu D, Geng J, Chen S, Jiang Z, et al. A modified multiplex ligation-dependent probe amplification method for the detection of 22q11.2 copy number variations in patients with congenital heart disease. BMC Genomics 2015;16:364.

25. Nothwang HG, Stubanus M, Adolphs J, Hanusch H, Vossmerbaumer U, Denich D, et al. Construction of a gene map of the nephronophthisis type 1 (NPHP1) region on human chromosome 2q12-q13. Genomics 1998;47:276-85.

26. Hildebrandt F, Singh-Sawhney I, Schnieders B, Papenfuss T, Brandis M. Refined genetic mapping of a gene for familial juvenile nephronophthisis (NPH1) and physical mapping of linked markers. APN Study Group. Genomics 1995;25:360-4.

27. Scolari F, Ghiggeri GM, Casari G, Amoroso A, Puzzer D, Caridi GL, et al. Autosomal dominant medullary cystic disease: a disorder with variable clinical pictures and exclusion of linkage with the NPH1 locus. Nephrol Dial Transplant 1998;13:2536-46.

28. Konrad M, Saunier S, Calado J, Gubler MC, Broyer M, Antignac C. Familial juvenile nephronophthisis. J Mol Med (Berl) 1998;76:310-6.

29. Exome Variant Server NGESPE. Seattle, WA (URL: http://evs. gs.washington.edu/EVS/).

30. Chaari I, Trabelsi M, Goucha R, Elaribi Y, Kharrat M, Guarguah T, et al. Prevalence and incidence estimation of large NPHP1 homozygous deletion in Tunisian population. Pathol Biol (Paris) 2012;60:e84-6.

31. Caridi G, Dagnino M, Gusmano R, Ginevri F, Murer L, Ghio L, et al. Clinical and molecular heterogeneity of juvenile nephronophthisis in Italy: insights from molecular screening. Am J Kidney Dis 2000;35:44-51.

32. Hildebrandt F, Otto E, Rensing C, Nothwang HG, Vollmer $M$, Adolphs J, et al. A novel gene encoding an SH3 domain protein is mutated in nephronophthisis type 1 . Nat Genet 1997;17:149-53.

33. Campbell IM, Yuan B, Robberecht C, Pfundt R, Szafranski P, McEntagart ME, et al. Parental somatic mosaicism is underrecognized and influences recurrence risk of genomic disorders. Am J Hum Genet 2014;95:173-82.

34. Dittwald P, Gambin T, Szafranski P, Li J, Amato S, Divon MY, et al. NAHR-mediated copy-number variants in a clinical population: mechanistic insights into both genomic disorders and Mendelizing traits. Genome Res 2013;23:1395-409.

35. Parisi MA, Bennett CL, Eckert ML, Dobyns WB, Gleeson JG, Shaw DW, et al. The NPHP1 gene deletion associated with juvenile nephronophthisis is present in a subset of individuals with Joubert syndrome. Am J Hum Genet 2004;75:82-91.

36. Roberts JL, Hovanes K, Dasouki M, Manzardo AM, Butler MG. Chromosomal microarray analysis of consecutive individuals with autism spectrum disorders or learning disability presenting for genetic services. Gene 2014;535:70-8. 